\title{
Perfil socioeducativo y económico de deportistas adolescentes de élite argentinos Socio-educational and economic profile of Argentinean elite adolescent athletes
}

\author{
Martin Gustavo Farinola, Ianina Tuñón, Fernando Laíño, Mario Marchesich, Martín Pérez Rodríguez
} Universidad Nacional de La Matanza (Argentina)

\begin{abstract}
Resumen. El objetivo de este estudio fue identificar si existen características socioeducativas y económicas que distinguen a los deportistas de élite adolescentes en Argentina. Se compararon los resultados obtenidos en los ítems correspondientes de la Encuesta de la Deuda Social Argentina en jóvenes de ambos sexos de 14 a 17 años de edad preseleccionados para los Juegos Olímpicos de la Juventud 2018 (n = 408), en jóvenes de población general que no realizan actividades físicas estructuradas extra-escolares $(n=1408)$ y en jóvenes que sí realizan estas actividades $(n=1086)$. Se encontraron diferencias significativas $(p<.05)$ en cuanto al tipo de hogar, nivel educativo de los padres, vivienda y acceso a bienes y servicios. Concluimos que los deportistas juveniles exitosos argentinos son diferentes y aventajados con respecto a la población general en cuanto a las dimensiones sociales aquí estudiadas. Estos resultados pueden ser de utilidad para futuros procesos de detección y desarrollo de jóvenes con potencial de éxito deportivo y apoyan la incorporación de enfoques multidimensionales para el estudio del desarrollo de la excepcionalidad deportiva.

Palabras clave: factores sociales, excepcionalidad deportiva, adolescentes, Juegos Olímpicos, Argentina.
\end{abstract}

\begin{abstract}
The aim of this study was to identify whether there are socio-educational and economic characteristics that distinguish elite adolescent athletes in Argentina. The results obtained in the corresponding items of the Encuesta de la Deuda Social Argentina were compared among young men and women between the ages of 14 and 17 who were shortlisted for the Youth Olympic Games 2018 (n = 408), i.e. young people from the general population who do not carry out structured out-of-school physical activities $(n=1408)$ and young people who do these activities $(n=1086)$. Significant differences $(p<.05)$ were found regarding the type of household, educational level of the parents, housing, and access to goods and services. We conclude that successful Argentine youth athletes are different and advantaged compared to the general population as regard to the social dimensions studied here. These results may be useful for future processes of detection and development of young people with potential for sport success and support the incorporation of multidimensional approaches to the study of the development of sports expertise.
\end{abstract}

Key words: social factors, sport expertise, adolescent, Olympic Games, Argentina.

\section{Introducción}

Mientras que los estudios clásicos sobre la excepcionalidad, o expertise, que se realizaron durante los siglos XIX y XX se basaron principalmente en la dicotomía nature-nurture(Baker \& Farrow, 2015a), estudios contemporáneos intentan identificar aquellos factores que se relacionan con la adquisición de la excepcionalidad deportiva, independientemente de si son innatos o adquiridos (Rees, et al., 2016). En este sentido proliferaron las investigaciones que buscaron responder el interrogante sobre qué es lo que caracteriza a los jóvenes deportistas que tuvieron éxito (Arias, 2008; Elferink-Gemser, Visscher, Lemmink \& Mulder, 2007; Marsillas, Rial, Isorna \& Alonso, 2014). Desde esta perspectiva teórica se han identificado factores relacionados con la excepcionalidad deportiva que provienen de diferentes niveles, tanto individuales como de contexto (Baker \& Farrow, 2015b; Bottoni, Gianfelici, Tamburri \& Faina, 2011). Los criterios para clasificar estos factores pueden variar. Baker y Horton (2004) los clasificaron como primarios (genéticos, de entrenamiento y psicológicos) y secundarios (socio-culturales y de contexto del deporte), dependiendo de sunivel de influencia sobre el rendimiento deportivo. Por su parte Rees et al. (2016) los clasificaron como factores del deportista (fecha de nacimiento, genéticos, fisiológicos y antropométricos, psicológicos), del entorno (lugar de nacimiento, apoyo familiar e institucional) y del entrenamiento (volumen de entrenamiento, presencia del juego, edad de especialización). Independientemente del sistema de clasificación de las influencias sobre la excepcionalidad deportiva es importante notar la naturaleza multidimensional de los nuevos esquemas de investigación sobre el tema (ver ejemplos en Arias, 2008 y en Bottoni, Gianfelici, Tamburri \& Faina, 2011).

Esta multidimensionalidad incluye a dimensiones sociales algunas de las cuales ya cuentan con evidencia de influencia significativa sobre el desarrollo de la excepcionalidad deportiva, como por ejemplo el apoyo familiar (Agudelo, et al., 2016; Kay, 2000; Velásquez, 2006). Otras de estas dimensiones, si bien todavía no cuentan con evidencia suficiente, los antecedentes disponibles permiten pensar que está justificado seguir estudiándolas, como por ejemplo el nivel socioeconómico familiar

Fecha recepción: 18-09-17. Fecha de aceptación: 20-12-17 Martin Gustavo Farinola martinfarinola@ymail.com
(Kay, 2000; Kirk, Carlson, O’Connor, Burke, Davis \& Glover, 1997; Rees, et al., 2016).

Estudios tempranos como el de Carlson (1988) sobre tenistas suecos mostraron que factores sociales como el ambiente en el club y la relación con los entrenadores, así como la estructura social y la tradición y cultura deportivas, eran factores que debían tenerse en cuenta además del talento individual para predecir la excepcionalidad deportiva. Yang, Telama y Laakso (1996) encontraron que la actividad física y la participación en deportes de jóvenes fineses, especialmente en los varones, estuvieron fuertemente relacionados a la práctica de actividad física de sus padres. En este estudio el nivel socio-económico de los padres no se asoció tan robustamente en los jóvenes varones aunque sí en el caso de las mujeres. Lo afirmado fue explicado como diferencias de género en cuanto a la expectativa social. Más adelante Kay (2000) encontró que el apoyo familiar era crucial para el desarrollo de jóvenes talentos deportivos en Gran Bretaña. Este apoyo consistía por un lado en la ayuda económica, pero por el otro en la adaptación de los horarios y agenda familiares a las obligaciones deportivas del joven. Esto permite conjeturar que el nivel socioeconómico familiar puede afectar el proceso de formación deportiva de los jóvenes potenciales expertos, al menos en Gran Bretaña. Resultados similares se encontraron recientemente en futbolistas españoles valencianos (Velásquez, 2006) y en deportistas escolares centroamericanos y caribeños (Agudelo, et al., 2016). Por su parte Velásquez (2008) realizó un estudio en futbolistas juveniles valencianos, España, donde comparó un grupo de jóvenes de alto nivel de rendimiento versus un grupo de jóvenes de menor nivel. Los resultados mostraron que no hubo diferencias entre los grupos en cuanto a variables sociales (p.ej. influencia familiar), pero sí las hubieron en cuanto a variables deportivas (p.ej. edad de inicio en el deporte o frecuencia semanal de entrenamiento). Esto puede indicar que las influencias sociales sobre los deportistas son características de los deportistas en su conjunto, independientemente del nivel deportivo que alcancen. Pero para corroborar esto se necesitará comparar al grupo de deportistas con la población general.

Hasta nuestro conocimiento un estudio que dé respuesta a estas cuestiones en deportistas de élite jóvenes argentinos está vacante. Por este motivo se aprovechará la oportunidad que ofrece la realización de los Juegos Olímpicos de la Juventud en la ciudad de Buenos Aires en 2018 para estudiar esta cuestión. 
Efectivamente, la tercera edición de los Juegos Olímpicos de la Juventud se llevará a cabo en Buenos Aires entre el seis y el 18 de octubre de 2018. La designación de esta ciudad como sede fue aprobada mediante votación por el Comité Olímpico Internacional el cuatro de julio de 2014, quedando Medellín(Colombia) en segundo lugar. En esta tercera edición se estima que participarán 3786 atletas de 15 a 18 años de edad, en 32 deportes diferentes. Los deportes son los mismos que en la edición anterior (Nankin 2014), con el agregado de: baile deportivo, skateboarding, patinaje sobre ruedas y escalada deportiva. Por otra parte, el fútbol fue reemplazado por fútbol sala.

En Argentina, desde la vigencia de la Ley 26573 (publicada en el Boletín Oficial el 22 de diciembre de 2009) se cuenta con el Ente Nacional deAlto Rendimiento Deportivo (ENARD), «como persona jurídica de derecho público no estatal destinado a gestionar y coordinar apoyos económicos específicos para la implementación y desarrollo de las políticas de alto rendimiento», tal como lo expresa el Artículo $\mathrm{N}^{\circ} 1$ del mencionado instrumento legal (Ministerio de Justicia y Derechos Humanos, 2009)

Desde el mismo momento de la confirmación de Buenos Aires como sede de la tercera edición de los Juegos Olímpicos de la Juventud, el ENARD asumió la responsabilidad de seleccionar y preparar a la delegación argentina. Así quedó expresado por el citado Ente, en su Informe para Gobiernos Provinciales y Federaciones Nacionales finalizado en 2014 y basado en una evaluación nacional de la aptitud física. En dicho informe se establece como punto de partida de la tarea para los Juegos la «identificación y selección de un grupo de jóvenes con facultades excepcionales y alto potencial para integrar el equipo nacional» (ENARD, 2015, p. 3)

A su vez, expresa que «cabe suponer que un joven talentoso manifiesta propiedades de base individual que superan el percentil 97 entre su grupo de pares» (ENARD, 2015, p. 4). Y la «identificación de estos jóvenes se vincula con el reconocimiento de capacidades diferenciadas para los distintos deportes, implicando algo más que una simple detección, ya que la conversión de dicha potencialidad en respuestas eficientes para la alta competencia, requiere un proceso de transformación en el que intervienen múltiples factores» (ENARD, 2015, p. 4).

Es importante destacar que para la identificación de estos jóvenes, desde el ENARD se plantearon tres objetivos operativos (ENARD, 2015):

1. Evaluación Nacional de la Aptitud Física, realizada en todo el territorio argentino y que recopiló información sobre aptitud física, hábitos e intereses deportivos entre 226019 jóvenes nacidos entre 2000 y 2001;

2. Búsqueda de deportistas en el sistema federativo, con registro y testeo de los 53037 deportistas federados, exceptuando el fútbol, con su consecuente segmentación y derivación por nivel.

3. Juegos Nacionales Evita 2014, mediante la observación de competencias y evaluaciones a los finalistas de pruebas consideradas clave.

Como principal conclusión del mencionado informe realizado por el ENARD, se destaca la detección de un grupo de más de 5000 deportistas con proyección olímpica que fueron evaluados en intervalos regulares para revalidar sus condiciones. Al momento de realizar la presente investigación se mantenían en el programa unos 530 atletas juveniles formando parte de un proceso intensivo de desarrollo deportivo con miras a su eventual participación en los Juegos Olímpicos de la Juventud 2018. Estos atletas fueron los más exitosos y de entre quienes se desprenderá el grupo que finalmente participe de los juegos.

Volviendo al interrogante sobre qué distingue a los jóvenes deportistas exitosos cabe preguntarse en qué medida estos jóvenes preseleccionados en el territorio de la Argentina es diferente del promedio de la población adolescente del país. Dentro de las múltiples dimensiones que afectan al desarrollo de la excepcionalidad deportiva este trabajo se dedicará a estudiar parte de los factores llamados secundarios (Baker \& Horton, 2004) o del entorno (Rees, et al., 2016), más precisamente el objetivo de este estudio es identificar si existen características socioeducativas y socioeconómicas que distingan a los jóvenes con proyección olímpica en Argentina.

\section{Material y Método}

\section{Participantes}

Se trabajó con dos fuentes de datos, una primaria y una secundaria respectivamente: (1) Una encuesta realizada en el primer trimestre de 2017 en el marco del ENARD a los deportistas preseleccionados para participar de los Juegos Olímpicos de la Juventud Buenos Aires 2018; y (2) la Encuesta de la Deuda Social Argentina (EDSA) del Programa del Observatorio de la Deuda Social Argentina de la Universidad Católica Argentina. La EDSA se realiza todos los años y para este trabajo se utilizaron los micro-datos de la EDSA correspondiente al tercer trimestre de 2016

El relevamiento realizado a los deportistas en el marco del ENARD se basó en una muestra de 408 jóvenes de 14 a 17 años de edad de ambos sexos sobre una población total de 530 atletas juveniles provenientes de las 24 provincias de Argentina y de 29 deportes que fueron preseleccionados para participar de los Juegos Olímpicos de la Juventud Buenos Aires 2018. Con algunas excepciones, la proporción de jóvenes de cada provincia en la muestra de deportistas es similar a la proporción de jóvenes de la misma edad en la población total. Por ejemplo la provincia y la ciudad de Buenos Aires son los distritos con mayor cantidad de jóvenes de 14 a 17 años de edad del país y son los distritos con más jóvenes de esa edad en la muestra de deportistas (Tabla 1).

Por el otro lado, en la EDSA la muestra fue estratificada en términos socioeconómicos a partir de una clasificación de conglomerados residenciales (unidades censales) según el perfil educativo predominante de los jefes de hogar. De esta manera, quedaron clasificados cinco espacios residenciales socioeducativos: Muy Bajo, Bajo, Medio Bajo, Medio y Medio Alto. En el universo geográfico de la EDSA, se consideran 20 aglomerados urbanos de 80000 habitantes y más: Área Metropolitana del Gran Buenos Aires (Ciudad Autónoma de Buenos Aires y partidos del Conurbano Bonaerense), Gran Córdoba, Gran Rosario, Gran Mendoza y San Rafael, Gran Salta, Gran Tucumán y Tafí Viejo, Mar del Plata, Gran Paraná, Gran San Juan, Gran Resistencia, NeuquénPlottier, Zárate, Goya, La Rioja, Comodoro Rivadavia, Ushuaia y Río Grande (Tuñón, 2017).

En el contexto del objetivo de este estudio, y para analizar la influencia de los factores sociales sobre la práctica deportiva, a esta muestra se la dividió en dos subgrupos: un grupo de adolescentes de entre 14 y 17 años de edad residentes en la Argentina urbana que no realizan actividad física estructurada extra-escolar $(\mathrm{n}=1408)$ y un grupo de adolescentes de entre 14 y 17 años de edad residentes en la Argentina urbana que realizan actividad física estructurada extra-escolar ( $\mathrm{n}=1086$ ). Para realizar esta agrupación se consideró a la actividad física estructurada extra-escolar como aquella que los adolescentes pueden desarrollar orientados por un profesor de Educación Física, en la práctica de algún

Tabla 1.

Porcentaje de jóvenes de 14 a 17 años de edad en la muestra de deportistas y en cada provincia con relación al total del país

\begin{tabular}{lcc} 
Con relación al total del país & Muestra & Total de la provincia* \\
\hline Provincia & $51,2 \%$ & $41,3 \%$ \\
\hline Buenos Aires y CABA &, $5 \%$ & $1,1 \%$ \\
Catamarca &, $5 \%$ & $3,3 \%$ \\
Chaco & $2,2 \%$ & $1,3 \%$ \\
Chubut & $8,7 \%$ & $7,9 \%$ \\
Córdoba & $1,5 \%$ & $3,0 \%$ \\
Corrientes & $2,7 \%$ & $3,2 \%$ \\
Entre Ríos &, $2 \%$ & $1,8 \%$ \\
Formosa &, $7 \%$ & $2,1 \%$ \\
Jujuy & $1,0 \%$ &, $8 \%$ \\
La Pampa &, $7 \%$ & $1,0 \%$ \\
La Rioja & $5,7 \%$ & $4,4 \%$ \\
Mendoza & $1,7 \%$ & $3,6 \%$ \\
Misiones & $1,7 \%$ & $1,5 \%$ \\
Neuquén & $4,0 \%$ & $1,7 \%$ \\
Río Negro & $1,5 \%$ & $3,8 \%$ \\
Salta & $2,0 \%$ & $1,8 \%$ \\
San Juan & $1,0 \%$ & $1,2 \%$ \\
San Luis &, $2 \%$ &, $7 \%$ \\
Santa Cruz & $9,7 \%$ & $7,5 \%$ \\
Santa Fe &, $2 \%$ & $2,7 \%$ \\
Santiago del Estero & $1,0 \%$ &, $3 \%$ \\
Tierra del Fuego & $1,0 \%$ & \\
Tucumán & & $4,0 \%$ \\
\hline CABA: Cindad Autón &
\end{tabular}

Tucumán

*Fuente: INDEC, 2010 
deporte u otro tipo de actividad física, que se desarrolle en clubes, gimnasios, escuelas (como actividad extracurricular), o en organizaciones barriales, como Sociedades de Fomento o similares (Tuñon \& Laíño, 2014).

\section{Instrumento}

La EDSA cuenta con un módulo específico destinado a medir el desarrollo humano y social de la niñez y adolescencia donde el informante es el adulto de referencia (madre, padre, principal cuidador) del joven entre 0 y 17 años (Tuñón, 2017). El instrumento de encuesta utilizado en el marco del relevamiento a los deportistas olímpicos incluyó un conjunto de indicadores originales de la EDSA en el campo de la educación de los padres, educación de los jóvenes, atributos de la vivienda, hogar y hábitat. Específicamente se hicieron las siguientes preguntas cerradas: asistís a la escuela, cómo se conforma el grupo familiar con el que actualmente convivís, cuántas personas en total contándote a vos mismo conviven en la misma vivienda, cuál es el máximo nivel educativo alcanzado por tu padre y por tu madre, tu vivienda tiene... (aquí se mencionan diversos bienes y servicios, ver Resultados).

\section{Procedimiento}

Luego de una presentación y explicación de los objetivos eítems de la encuesta, los jóvenes deportistas olímpicos fueron encuestados de manera autoadministrada. Se trabajó en grupos de deportistas con dos coordinadores de encuesta presentes para evacuar posibles dudas que les puedan surgir a los respondientes. La administración del instrumento se realizó entre el jueves dos al domingo 12 de febrero de 2017 en las instalaciones del Centro Nacional de Alto Rendimiento Deportivo (CeNARD) durante el llamado Campus 2018 organizado por el ENARD. La realización de la encuesta formó parte de las actividades previstas para el campus y contó con la aprobación y el apoyo tanto de los funcionarios del ENARD como de los entrenadores de las selecciones de los diferentes deportes. La participación en el estudio por parte de los jóvenes fue anónima y voluntaria.

\section{Análisis de los datos}

El análisis que se realizó es de tipo descriptivo con base en distribuciones porcentuales para cada uno de los tres grupos de comparación en el conjunto de indicadores socioeducativos y socioeconómicos correspondientes. Luego se realizaron contrastes estadísticos mediante el test U de Mann-Whitney para muestras independientes con el objetivo de establecer si las diferencias porcentuales entre los grupos de referencia son significativas. La significación estadística se estableció para $p<.05$. El tratamiento estadístico fue realizado con el software IBM SPSS ${ }^{\circledR}$ Statistics versión 23.0 (IBM Corp., Armor, New York).

\section{Resultados}

A partir de la EDSA se estima que en 2016 alrededor del 43\% de los jóvenes entre 14y 17 años realizaba alguna actividad física estructurada extra-escolar. La propensión a realizar deportes o actividad física extraescolar en este grupo de edad deportistas amateur es mayor en los varones que en las mujeres. Esta distribución según el sexo es muy similar a lo que ocurre en el interior de la población de adolescentes que están en el proceso de selección para participar de los Juegos Olímpicos de la Juventud Buenos Aires 2018 (véase Tabla 2).

Con respecto al tipo de configuración de las familias lo que se observa es que los deportistas olímpicos registran mayor propensión que los otros dos grupos a pertenecer a hogares biparentales conformados por dos cónyuges. Es decir, que presentan menos probabilidad de que sus hogares sean monoparentales con un núcleo con presencia de un solo cónyuge (véase Tabla 2).

La mayoría de los adolescentes asisten a la escuela formal entre los 14 y 17 años en la Argentina. No obstante, en la comparación entre grupos se advierte que los deportistas olímpicos registran menor propensión a la no escolarización que los grupos de comparación.

El perfil socioeducativo familiar de los adolescentes que no realizan actividades físicas estructuradas extra-escolares en la Argentina urbana indica que más del $60 \%$ pertenecen a hogares de bajo clima educativo (64\% son hijos de padre con secundario incompleto o menos), mientras que en los adolescentes que sí realizan actividades físicas estructuradas extra-escolares a nivel de la población en general este porcentaje es menor (48,7\%). No obstante, los adolescentes en proceso de selección para participar de los Juegos Olímpicos presentan un perfil socioeducativo muy diferente, en efecto, $20,8 \%$ pertenece a hogares cuyo padre tiene estudios secundarios incompletos o menos (véase Tabla 2). Cuando se considera el nivel educativo de la madre o referente mujer de los hogares las diferencias siguen la misma tendencia observada en el caso de la educación de los padres o referente adulto varón. Esto es, el nivel educativo se incrementa en los grupos de deportistas amateur y más aún en el grupo de los deportistas olímpicos.

Otros atributos que permiten confirmar la posición relativa de ventaja socioeconómica de los adolescentes olímpicos respecto de pares no deportistas y pares deportistas amateur son: el acceso a servicios como red de cloacas, inodoro con descarga, red eléctrica, red de agua, y otros menos esenciales como tener computadora o servicio de internet o cable en la vivienda. Si bien, tal como se puede advertir en la Tabla 2, los adolescentes que realizan deportes amateur tienen niveles de privaciones menos elevados que los observados a nivel de la población de no deportistas, las carencias en la población de deportistas olímpicos son casi residuales (véase Tabla 2). Esta tendencia de relativa ventaja

Tabla 2.

Perfil socioeducativo y socioeconómico de tres grupos poblacionales en porcentaje de jóvenes entre 14 y 17 años de edad

\begin{tabular}{|c|c|c|c|c|c|c|c|}
\hline \multirow[b]{2}{*}{ Variables } & \multirow[b]{2}{*}{ Categorías } & \multicolumn{3}{|c|}{ Grupos de comparación } & \multicolumn{3}{|c|}{ Valor $p$} \\
\hline & & Población general (1) & Deportistas amateur (2) & Deportistas Olímpicos (3) & Grupo 1 y 2 & Grupo 1 y 3 & Grupo 2 y 3 \\
\hline \multirow{2}{*}{ Sexo } & Varón & $44,1 \%$ & $61,5 \%$ & $61,0 \%$ & \multirow{2}{*}{$.000^{*}$} & \multirow{2}{*}{$.000^{*}$} & \multirow{2}{*}{.945} \\
\hline & Mujer & $55,8 \%$ & $38,4 \%$ & $38,9 \%$ & & & \\
\hline Tipo de hogar & $\begin{array}{l}\text { Hogar biparental } \\
\text { Hogar monoparental }\end{array}$ & $\begin{array}{l}74,8 \% \\
25,2 \%\end{array}$ & $\begin{array}{l}75,4 \% \\
24,5 \%\end{array}$ & $\begin{array}{l}84,3 \% \\
15,6 \%\end{array}$ & .752 & $.000^{*}$ & $.000^{*}$ \\
\hline Escolarización & $\begin{array}{l}\text { Asiste } \\
\text { No asiste }\end{array}$ & $\begin{array}{l}92,7 \% \\
7,2 \%\end{array}$ & $\begin{array}{c}95,8 \% \\
4,1 \%\end{array}$ & $\begin{array}{l}97,2 \% \\
2,7 \%\end{array}$ & $.004 *$ & $.003^{*}$ & .240 \\
\hline \multirow{6}{*}{ Máximo nivel educativo del padre } & Primario incompleto & $10,5 \%$ & $5,7 \%$ & $2,9 \%$ & $.000^{*}$ & $.000^{*}$ & $.016^{*}$ \\
\hline & Primario completo & $36,6 \%$ & $25,6 \%$ & $4,4 \%$ & $.000^{*}$ & $.000^{*}$ & $.000^{*}$ \\
\hline & Secundario incompleto & $16,9 \%$ & $16,7 \%$ & $13,4 \%$ & .947 & .110 & .110 \\
\hline & Secundario completo & $25,3 \%$ & $30,4 \%$ & $19,6 \%$ & $.031^{*}$ & $.007 *$ & $.000^{*}$ \\
\hline & Terciario/Univ. incompleto & $2,3 \%$ & $4,3 \%$ & $21,3 \%$ & $.002^{*}$ & $.000^{*}$ & $.000^{*}$ \\
\hline & Terciario/Univ. completo & $8,1 \%$ & $17,1 \%$ & $38,0 \%$ & $.000^{*}$ & $.000^{*}$ & $.000^{*}$ \\
\hline \multirow{6}{*}{ Máximo nivel educativo de la madre } & Primario incompleto & $9,0 \%$ & $3,8 \%$ & $1,2 \%$ & $.000^{*}$ & $.000^{*}$ & .004 \\
\hline & Primario completo & $34,3 \%$ & $21,0 \%$ & $2,2 \%$ & $.000^{*}$ & $.000^{*}$ & $.000^{*}$ \\
\hline & Secundario incompleto & $16,4 \%$ & $20,1 \%$ & $9,1 \%$ & $.003^{*}$ & $.000^{*}$ & $.000^{*}$ \\
\hline & Secundario completo & $26,9 \%$ & $29,8 \%$ & $19,0 \%$ & .590 & $.000^{*}$ & $.000^{*}$ \\
\hline & Terciario/Univ. incompleto & $3,5 \%$ & $7,0 \%$ & $20,0 \%$ & $.000^{*}$ & $.000^{*}$ & $.000^{*}$ \\
\hline & Terciario/Univ. completo & $9,6 \%$ & $18,0 \%$ & $48,4 \%$ & $.000^{*}$ & $.000^{*}$ & $.000^{*}$ \\
\hline \multirow{3}{*}{ Indicadores de déficit en acceso a bienes y servicios } & No tener ordenador & $36,3 \%$ & $21,7 \%$ & $5,7 \%$ & $.000^{*}$ & $.000^{*}$ & $.000^{*}$ \\
\hline & No tener internet & $46,8 \%$ & $33,4 \%$ & $4,2 \%$ & $.000^{*}$ & $.000^{*}$ & $.000^{*}$ \\
\hline & No tener cable de TV & $29,4 \%$ & $19,2 \%$ & $6,0 \%$ & $.000^{*}$ & $.000^{*}$ & $.000^{*}$ \\
\hline \multirow{4}{*}{ Indicadores de déficit en el espacio de la vivienda } & Hacinamiento & $22,4 \%$ & $9,9 \%$ & $10,2 \%$ & $.000^{*}$ & $.000^{*}$ & .921 \\
\hline & No acceder a la red de agua & $15,8 \%$ & $15,9 \%$ & $3,6 \%$ & .993 & $.000^{*}$ & $.000^{*}$ \\
\hline & No tener retrete con descarga & $17,3 \%$ & $6,7 \%$ & $1,7 \%$ & $.000^{*}$ & $.000^{*}$ & $.000^{*}$ \\
\hline & No tener cloacas & $44,6 \%$ & $37,6 \%$ & $10,0 \%$ & $.002 *$ & $.000^{*}$ & $.000^{*}$ \\
\hline
\end{tabular}


socioeconómica de la población de deportistas olímpicos también se puede advertir en el acceso a bienes y servicios de los hogares como ordenador, servicio de internet y de cable para la televisión. Asimismo, en las características básicas de las viviendas en que residen (acceso al agua de red, inodoro con descarga, entre otros).

Se evidencia que los adolescentes deportistas olímpicos pertenecen a hogares con un elevado clima educativo y de inserción social aventajada respecto de otros grupos de adolescentes. Si bien, los adolescentes que realizan actividades físicas estructuradas extra-escolares de modo amateur tienen una posición socioeducativa y social algo mejor que la población adolescente que no realiza este tipo de actividades extraescolares, los adolescentes deportistas olímpicos pertenecen en promedio a hogares de estratos medios y medios altos.

\section{Discusión}

La pregunta acerca de qué es lo que caracteriza a los deportistas juveniles que tienen éxito se puede responder si se comparan a estos deportistas con población general de las mismas características en cuanto a país, época y edad (Arias, 2008). Para este trabajo se ha tenido la oportunidad de realizar esta comparación al contar con información confiable de tres poblaciones (población juvenil que no realiza actividades físicas estructuradas extra-escolares, pares que lo hacen de modo amateur y los deportistas juveniles olímpicos) y de esta forma poder comenzar a dar respuesta a este interrogante para el caso de la Argentina.

En cuanto al apoyo familiar, según Kay (2000) y Kirk (2005), se concreta al menos de dos maneras; por un lado acomodando sus agendas para poder acompañar al joven a las actividades que el deporte demande y por el otro otorgando los recursos económicos que su formación como deportista requieran. Aquí hemos encontrado que los jóvenes deportistas exitosos argentinos se caracterizan por tener mayor presencia en el hogar tanto del padre como de la madre en el marco de configuraciones familiares biparentales constituidas por ambos referentes adultos. En este espacio del desarrollo de capacidades en la infancia y adolescencia como en otros la configuración familiar es clave. Existen estudios que señalan la debilidad de los hogares monoparentales en los procesos de socialización en edades infantiles y juveniles frente a los biparentales (Montoya \& Landero, 2008; Tuñón, 2010). Más adultos de referencia presentes posibilitan un mejor acompañamiento en el desarrollo humano y social. En efecto, la presencia de los adultos de referencia en el hogar puede afectar de modo positivo el desarrollo deportivo de los jóvenes debido a que son los principales agentes socializadores y los más influyentes en la iniciación deportiva (Agudelo, et al., 2016; Arias, 2008) y son quienes ayudan a mitigar las presiones que ocurren durante el deporte competitivo a medida que los jóvenes van creciendo (Baker \& Horton, 2004; Chamorro, Torregrosa, SánchezMiguel, Sánchez-Oliva, \&Amado, 2015).

Con respecto al nivel socioeconómico familiar hemos encontrado mayores diferencias entre los grupos que en la variable anterior. Por un lado tanto en el padre como en la madre hubo diferencias notorias y significativas de nivel educativo, siendo aproximadamente tres veces mayor el terciario o universitario completo y tres veces menor la primaria incompleta en los padres de los deportistas olímpicos con respecto a los de población general. Por lo tanto se advierte que los deportistas olímpicos pertenecen a hogares de mayor clima educativo que la población general, y que a medida que aumenta el clima educativo de los hogares se incrementa la probabilidad de especialización de los adolescentes en el campo del deporte. $\mathrm{Al}$ analizar de manera discriminada al grupo de población general emerge que en el grupo de jóvenes que practican actividades físicas estructuradas extra-escolares los valores son intermedios con respecto a los otros dos grupos, dándole fuerza a la idea de la influencia del nivel educativo de los padres sobre la práctica deportiva de los jóvenes. Más específicamente, los adolescentes que realizan actividades físicas estructuradas extra-escolares pertenecen a hogares con mejor clima educativo que la media de sus pares que no realizan estas actividades, sin embargo los adolescentes preseleccionados en el marco de los Juegos Olímpicos se diferencian de modo significati- vo y pertenecen en una amplia mayoría a hogares cuyo padre o madre tiene estudios secundarios completos o más, es decir a hogares con un elevado clima educativo.

Por otro lado, en todas las dimensiones de bienes y servicios también hubo diferencias notorias y significativas entre los grupos. Prácticamente no hubo jóvenes deportistas olímpicos que carezcan de ellos (todas las dimensiones excepto una estuvieron en un dígito), mientras que en población general de no deportistas hubieron dimensiones que llegaron a superar el $40 \%$ de carencia. Es interesante observar que, al igual que en las variables educativas familiares, en el grupo de población general que realiza actividades físicas estructuradas extra-escolares se encontraron valores intermedios, mostrando asociación entre estas dimensiones del nivel socioeconómico y la práctica deportiva.

El nivel socioeconómico familiar es un tema que se ha sugerido recientemente debe estudiarse en mayor profundidad como factor potencial para el desarrollo del rendimiento deportivo juvenil (Rees, et al., 2016) debido al resultado obtenido en los estudios realizados hasta el momento (Kay, 2000; Kirk, et al., 1997; Yang, Telama \& Laakso, 1996), nuestros resultados apoyan esta sugerencia. Como se señaló, se ha encontrado una diferencia significativa en cuanto a esta dimensión en atletas exitosos versus población de no deportistas y practicantes amateur, lo que puede indicar cierto efecto sobre el desarrollo de la excepcionalidad deportiva. Si bien este ha sido un estudio transversal y no es posible adjudicar causalidad, existen razones para creer que hay algún nivel de efecto del nivel socioeconómico sobre el desarrollo de la excepcionalidad deportiva. Según Kirk (2005) la participación en deportes requiere de recursos para solventar cuotas, equipamiento y transporte a los entrenamientos y las competencias, y por otro lado las familias con horarios flexibles se encuentran en mejores condiciones para el acompañamiento de los jóvenes, sobre todo cuando se trata de más de un hijo. Esto afecta más fuertemente al deporte de élite, en el cual los compromisos de los jóvenes son mayores e incluso pueden incluir viajes internacionales por varios días. Pero también afectaría al deporte amateur. Velásquez (2008) no encontró diferencias significativas en variables sociales entre deportistas jóvenes de élite versus los de menor nivel. Aquí hemos encontrado diferencias pero las mismas han sido menores entre los jóvenes deportistas de élite y los practicantes de menor nivel que con los jóvenes de población general que no realizan actividades físicas estructuradas extra-escolares. Esto muestra una similitud y una diferencia con el estudio de Velásquez (2008). Por un lado las influencias sociales sobre la práctica existen independientemente del nivel que alcancen los jóvenes, pero por el otro estas influencias son mayores cuanto mayor es el compromiso que se requiere con la práctica deportiva.

Estos resultados, de corroborarse, pueden además llamar la atención de los encargados de identificar y desarrollar la excepcionalidad deportiva de jóvenes con proyección de éxito. Pese a que en el programa del ENARD la detección de talentos se realizó en todo el territorio del país con la vocación de llegar con los recursos del Estado a los lugares más recónditos, y que se testeó a más de 200000 jóvenes de ambos sexos (ENARD, 2015), la evidencia presentada en este artículo sugiere lo que la literatura disponible sobre la cuestión viene señalando acerca de la mayor probabilidad que tienen los jóvenes de estratos medios y medios altos de desarrollar la excepcionalidad deportiva. Estos resultados contribuyen a comprender que los esfuerzos que realizan los Estados en países como la Argentina requieren ser sistemáticos en el acompañamiento de poblaciones vulnerables en las que probablemente existe un enorme potencial de desarrollo que no logra visibilidad debido a que los recursos de los hogares no son suficientes para lograr apropiarse de las estructuras de oportunidades vigentes en el país o porque incluso las mismas no están al alcance de estas poblaciones y carecen de los recursos necesarios para sostener, estimular y promover a jóvenes deportistas en situación de desventaja social.

Uno de los puntos fuertes de este estudio fue la representatividad de las muestras y haber utilizado las mismas preguntas en los diferentes grupos, lo que vuelve más robusta la comparación realizada. Una limitación del estudio fue no haber encuestado a la totalidad de los deportis- 
tas olímpicos que participaron del Campus 2018, esto se dificultó por razones ajenas al equipo deinvestigación. Sin embargo sellegó a encuestar a una porción importante de los mismos (77\%) y esta porción no fue elegida deliberadamente por los investigadores, lo cual refleja cierta aleatoriedad en la selección de sujetos que elimina posibles sesgos de los investigadores.

Futuras investigaciones se necesitan para identificar en detalle cuáles son las dimensiones sociales que más influyen sobre el desarrollo de la excepcionalidad deportiva en Argentina y propender a la construcción de un modelo de identificación y desarrollo eficaz de esta excepcionalidad.

\section{Conclusiones}

Concluimos que los deportistas juveniles exitosos argentinos son diferentes a la población general en cuanto a las dimensiones sociales aquí estudiadas. Se caracterizan por tener mayor presencia de ambos padres o adultos de referencia en el hogar, mayor nivel educativo de los mimos y mayor acceso a bienes y servicios. Se trata de una población de adolescentes aventajados en términos del clima socioeducativo de los hogares, el tipo de configuración familiar mayormente de núcleo conyugal completo y de nivel socioeconómico medio alto.

Estos resultados pueden ser de suma utilidad para futuros procesos de identificación y desarrollo de la excepcionalidad deportiva en jóvenes con proyección de éxito señalando la necesidad de acompañamiento de poblaciones vulnerables en las que probablemente existe un enorme potencial deportivo que no logra visibilidad y que cuenta con insuficientes recursos y posibilidades de acceso y desarrollo en relación con las demandas y requerimientos que exige el deporte de élite.

Por último, y al igual que lo concluido en otros trabajos (Arias, 2008; Bottoni, Gianfelici, Tamburri \& Faina, 2011), los resultados de este estudio aportan evidencia sobre la importancia de continuar realizando estudios desde enfoques multidimensionales del desarrollo de la excepcionalidad deportiva.

\section{Agradecimientos}

Este artículo se desarrolló en el marco del Proyecto PROINCE 55A206 «Factores multidimensionales asociados al desarrollo de potenciales atletas de élite seleccionados para los Juegos Olímpicos de la Juventud 2018», de la Facultad de Ciencias Sociales de la Universidad Nacional de la Matanza, Argentina, y bajo la dirección de Ianina Tuñón. Se agradece la colaboración del equipo que realizó el relevamiento de datos: Hernán Castro, Brígida Lavignole y Franco Albino Rodríguez, además de los autores. Un agradecimiento especial al Ente Nacional de Alto Rendimiento Deportivo (ENARD). Los autores además agradecen al Observatorio de la Deuda Social Argentina de la Universidad Católica Argentina el acceso a los micro-datos de la Encuesta de la Deuda Social Argentina.

\section{Referencias}

Agudelo, A.; Ayala, C.; Ramos, S.; García, A.; Melo, L.; Aguirre-Loaiza, H.; ... Martínez, K. (2016). Características de la formación deportiva de los participantes en IV Juegos Escolares Centroamericanos y del Caribe, Armenia 2013. Lúdica Pedagógica, 1(23), 127-139.

Arias, J. (2008). El proceso de formación deportiva en la iniciación a los deportes colectivos fundamentado en las características del deportista experto. Retos. Nuevas tendencias en Educación Física, Deporte y Recreación, 13, 28-32.

Baker, J., \& Farrow, D. (2015a). A [very brief] review of the historical foundations of sport expertise: An introduction to the Handbook. En J. Baker, D. Farrow. (Eds.), Routledge Handbook of Sport Expertise (pp. 1-8). Abingdon: Routledge.

Baker, J., \& Farrow, D. (Eds.).(2015b). Routledge Handbook of Sport Expertise. Abingdon: Routledge.

Baker, J., \& Horton, S. (2004). A review of primary and secondary inûuences on sport expertise. High Ability Studies, 15(2), 211-226.

Bottoni, A.; Gianfelici, A.; Tamburri, R., \& Faina, M. (2011). Talent selection criteria for olympic distance triathlon. Journal of Human Sport and Exercise, 6(2), 293-304.

Carlson, R. (1988). The socialization of elite tennis players in Sweden: An analysis of the players' backgrounds and development. Sociology of Sport Journal, 5(3), 241-256.

Chamorro, J.; Torregrosa, M., Sánchez-Miguel, P.; Sánchez-Oliva, D., \& Amado, D. (2015). Desafíos en la transición a la élite del fútbol: recursos de afrontamiento en chicos y chicas. Revista Iberoamericana de Psicología del Ejercicio y el Deporte, 10(1), 113-119.

Elferink-Gemser, M.; Visscher, C.; Lemmink, K., \& Mulder, T. (2007). Multidimensional performance characteristics and standard of performance in talented youth field hockey players: A longitudinal study. Journal of Sports Sciences, 25(4), 481-489.

Ente Nacional de Alto Rendimiento Deportivo. (2015). Evaluación nacional de aptitud física 2014. Informe para Gobiernos Provinciales y Federaciones Nacionales. ENARD. Buenos Aires. (No publicado).

INDEC (Instituto Nacional de Estadística y Censos) (2010). Censo Nacional de Población, Hogares y Viviendas 2010. Recuperado de https:/

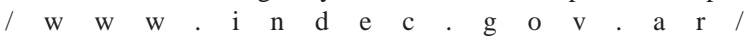
nivel4_default.asp?id_tema_1=2\&id_tema_2=41\&id_tema_3=135

Kay, T. (2000). Sporting excellence: A family affair? European Physical Education Review, 6(2):151-169.

Kirk, D. (2005). Physical education, youth sport and lifelong participation: the importance of early learning experiences. European Physical Education Review, 11(3), 239-255.

Kirk, D.; Carlson, T.; O’Connor, A.; Burke, P.; Davis, K., \& Glover, S. (1997). The economic impact on families of children's participation in junior sport. Australian Journal of Science and Medicine in Sport, 29(2), 27-33.

Marsillas, S.; Rial, A.; Isorna, M., \& Alonso, D. (2014). Niveles de rendimiento y factores psicológicos en deportistas en formación. Reflexiones para entender la exigencia psicológica del alto rendimiento. Revista Iberoamericana de Psicología del Ejercicio y el Deporte, 9(2), 373-392.

Ministerio de Justicia y Derechos Humanos. (2009). Ley 26573:Ente Nacional de Alto Rendimiento Deportivo, Creación. Boletín Oficial del 22-dic-2009, Número 31806, Página 3. Recuperado de http:// servicios.infoleg.gob.ar/infolegInternet/anexos/160000-164999/ 161877/norma.htm

Montoya, B., \& Landero, R. (2008). Satisfacción con la vida y autoestima en jóvenes de familia monoparentales y biparentales. Revista Psicología y Salud, 18(1), 117- 122.

Rees, T.; Hardy, L.; Güllich, A.; Abernethy, B.; Côté, J.; Woodman, T.; ..., Warr, C. (2016). The Great British Medalists Project: A review of current knowledge on the development of the world's best sporting talent. Sports Medicine, 46(8), 1041-1058.

Tuñón, I. (2010). Determinantes de las oportunidades de crianza y socialización en la niñez y en la adolescencia. Revista Latinoamericana de Ciencias Sociales, Niñez y Juventud, 8(2), 903-920.

Tuñón, I. (2017). Evolución de indicadores de desarrollo humano y social en la infancia en perspectiva de derechos humanos (20102016). Documento estadístico. Observatorio de la Deuda Social Argentina. Universidad Católica Argentina. Buenos Aires: Educa. Recuperado de http://www.uca.edu.ar/uca/common/grupo68/files/2017-Observatorio-Evolucion-indicadores-desarrollo-humano-y-social.pdf

Tuñón, I., \& Laíño, F. (2014). Insuficiente actividad física en la infancia: niños, niñas y adolescentes entre 5 y 17 años en la Argentina urbana. Observatorio de la Deuda Social Argentina. Universidad Católica Argentina. Buenos Aires: Educa. Recuperado de http://www.uca.edu.ar/ uca/common/grupo68/files/2014-Observatorio-Boletin-2-BDSI.pdf

Velásquez, R. (2006). Dimensiones sociales y ambientales que influyen en la trayectoria de futbolistas juveniles. PubliCE Standard. Recuperado de https://g-se.com/es/psicologia-del-deporte/articulos/dimensiones-sociales-y-ambientalesque-in-uyen-en-la-trayectoria-defutbolistas-juveniles-793

Velásquez, R. (2008). Análisis de los factores socio-deportivos y ambientales que intervienen en la trayectoria de futbolistas juveniles. PubliCE Premium. Recuperado de https://g-se.com/es/psicologia-del-deporte/ articulos/analisis-de-los-factores-socio-deportivos-y-ambientales-queintervienen-en-la-trayectoria-de-futbolistas-juveniles-974

Yang, X.; Telama, R., \& Laakso, L. (1996). Parents’ physical activity, socioeconomic status and education as predictors of physical activity and sport among children and youths: A 12-year follow-up study. International Review for the Sociology of Sport, 31(3), 273-291. 